\title{
BRAF V600E Mutation Present
}

National Cancer Institute

\section{Source}

National Cancer Institute. BRAF V600E Mutation Present. NCI Thesaurus. Code C80275.

A molecular finding indicating the presence of a BRAF V600E mutation in a tissue sample. 